\title{
Explorando luz solar en modelos de desarrollo inmobiliario. Aplicaciones en cinco ciudades chilenas.
}

Exploring sunlight in real estate models. Application on five Chilean cities.

Luz Alicia Cárdenas Jirón; Juan Pablo Vásquez Palau, Juan Carlos Zamorano, Claudia Acevedo

\section{Filiación}

Departamento de Urbanismo F.A.U. Universidad de Chile, arquitecto independiente, arquitecto independiente, F.A.U Universidad de Chile.

E mail: Icardena@uchilefau.cl

Primera versión recibida en: 28 de abril, 2016

Última versión recibida en: 25 de junio, 2016

\section{Resumen}

El explosivo crecimiento del stock edificado de los últimos años en las ciudades chilenas se ha visto reflejado en concentraciones inmobiliarias de gran altura, que irrumpen estrepitosamente en la morfología urbana. Externalidades son observadas en los espacios y edificios vecinos tales como extensas áreas de sombreamiento que afectan las condiciones térmicas y lumínicas. Estas concentraciones se realizan acordes a la norma urbanística, pero afectan la luz solar incidente en fachadas y suelos, disminuyendo las posibilidades de captación y uso como energía renovable limpia: factor relevante en contexto actual de Net Billing. El objetivo del presente artículo es explorar mediante modelos espaciales 3D y simulaciones computacionales energéticas, distintas formas de ocupación de suelo; cambiando la altura edificada, pero manteniendo la constructibilidad en cinco ciudades chilenas: Santiago, Valparaíso, Concepción, Temuco y Puerto Montt Las ciudades seleccionadas se sitúan en el centro sur de Chile, emplazadas tanto en el borde costero como el valle central y han presentado crecimientos inmobiliarios importantes en los últimos años. Los resultados muestran valores de energía capturada en fachadas mayores que en suelos y en configuraciones de más altura, respecto a las menores. Ello propone una discusión a la normativa actual chilena respecto a privilegiar la captación de luz solar en ámbito privado (fachadas) o en espacio público (suelos), o en ambos.

\section{Palabras clave}

Luz solar; norma urbanística; inmobiliaria; net billing, forma urbana.

\begin{abstract}
Explosive growing of building stock in the last years on the Chilean cities has been reflected on skyscrapers real states nodes that burst urban form. Externalities are observed as shadows that impact on thermal and luminous conditions above neighbourhood buildings and urban space. Those buildings have been constructed according to urbanism normative but they affect incident sunlight on facades and ground. Consequently it decreases possibilities to capture and use as clean renewable energy, relevant issue in current context of Net Billing. The objective of the paper is to explore different ways of plot site changing building height but keeping built coefficient in five Chilean cities: Santiago, Valparaíso, Concepción, Temuco, and Puerto Montt. Methodology consists of computational 3D modelling and energy simulation of spatial configuration. Cities selected are located on waterfront or on central valley in the middle of the Chilean territory in which there are the most urbanized areas with significant real state growing. Results shows higher energy values on facades of spatial configuration with high buildings and lower ones on ground. Thus a discussion is highlighted whether current urban ordinance should promote sunlight capture on private (facades) or public space (ground) or simply both of them.
\end{abstract}

Key words

Sunlight; urbanism regulations; real state; net billing, urban form 


\section{Sumario}

Introducción

1 Planificación urbana, norma urbanística y energética chilena asociada al inmobiliario residencial.

2 Metodología

3 Simulación energética en cinco ciudades chilenas

4 Autosuficiencia energética y la Generación Distribuida

5 Discusión y conclusiones

Bibliografía

\section{I ntroducción}

El explosivo crecimiento del stock edificado de los últimos años en las ciudades chilenas se ha visto reflejado en concentraciones inmobiliarias de gran altura, que irrumpen estrepitosamente en la morfología de las ciudades chilenas. (Daher, 2013; De Mattos, 2012, 2002; Galetovic, 2006). Figura 1. El mercado inmobiliario en su búsqueda por maximizar el valor de la inversión, puede aumentar la cabida de terrenos y su capacidad edificatoria a través de la Fusión de Terrenos que es permitida por la Ley General de Urbanismo y Construcciones en su $\operatorname{Art}^{\circ} 63$, aumentando la constructibilidad del predio en un 30\% (Becar, 2013). Más aún, Normas de Excepción como la Fusión de Terrenos permiten sobrepasar los máximos permitidos por los Planes Reguladores Comunales produciendo conflictos urbanos en los vecinos afectados y distorsiones a la planificación urbana (Alcaíno, 2008). Densidades con edificios en altura producen externalidades en los espacios y edificios cercanos tales como áreas de sombreamiento que afectan no solo el valor de las propiedades vecinas, sino que también las condiciones térmicas y lumínicas de los edificios. (Ratti et al., 2005; Hachem et al., 2014; Cárdenas y Vilches, 2015). Lo que se conoce como el factor de horizonte urbano (Baker and Steemers, 2000;). Adicionalmente, existe una creciente necesidad por fomentar el empleo de energías limpias ERNC ${ }^{1}$ en la matriz energética junto con inyectar el excedente de una autogeneración a la red pública, conocido como Ley Billing o Generación Distribuida (SEC, 2014). Una aparente contradicción surge si se desea fomentar la energía solar a partir de unidades residenciales y simultáneamente permitir densidades edificatorias en altura con los consecuentes conos de sombra proyectados por esas intervenciones arquitectónicas. En ese contexto, surgen interrogantes:

¿Qué ocurriría si el desarrollo inmobiliario permitiera a los edificios del entorno urbano la captación de luz solar y que ésta fuera una premisa de diseño arquitectónico-urbanístico?

¿Qué implicancias tienen las formas de ocupación de la norma urbanística en la promoción de la Ley Net Billing?

Para responder a las preguntas se definen objetivos que enmarcan el alcance de este artículo. Como objetivo general se propone explorar la incidencia de la luz solar en configuraciones espaciales de alta densidad observadas en los desarrollos inmobiliarios, comparando su comportamiento en diversas ciudades chilenas. Como objetivo específico se propone analizar la relación la forma de ocupación de suelo y la aplicación de Net Billing, a través del cruce de variables altura edificada y autoconsumo residencial.

\footnotetext{
${ }^{1}$ ERNC Las Energías Renovables No Convencional en Chile reconocidas por la Ley 20257 y Ley 20698 son las siguientes: eólica, solar, geotermia, bioenergía (biomasa o biogás), mini-hidráulica y mareomotriz. Reporte CIFES, 2015. Comité CORFO. Ministerio de Energía. Santiago de Chile.
} 
Las investigaciones de forma urbana, luz solar y normativas han sido largamente estudiadas tanto por los beneficios para la salud humana como por la generación de energía solar fotovoltaica o térmica. Knowles, uno de los pioneros arquitectos investigadores en el tema, establece que el sol es fundamental para la vida humana porque representa la fuente de energía que regula los ritmos de la vida, siendo la trayectoria solar un elemento que comunica perceptualmente al ser humano con la escala de tiempo y espacio. El clásico estudio sobre la arquitectura del pueblo de Acoma en América del Norte muestra la adaptación a un clima extremo desértico, analizando distanciamientos y alturas como maneras básicas de planificación solar vernacular (Knowles, 2003: 15).

Este autor desarrolla el concepto de envolvente solar para ser aplicado a las ciudades con el fin de garantizar el acceso de luz solar a las edificaciones. Afirma que a los desarrolladores no les preocupa el costo energético en el largo plazo de los edificios para que sean confortables y alcanzables las facturas de calefacción, refrigeración e iluminación. Al contrario, el rápido crecimiento demanda a los arquitectos especificar con sistemas intensivos en energía en vez de diseñar con la naturaleza. En términos simples, "crecer rápido" y "mantener caro". Resulta de interés notar que estos procesos de crecimiento urbano rápido presentado por los desarrolladores inmobiliarios, ignorando las condiciones espaciales favorables a la disponibilidad de energía solar, también ocurren en Chile.

El envolvente solar es un constructo espacio-tiempo basado en la trayectoria solar que aplica en calles, manzanas y edificios para relevar la importancia que tiene considerarlo en la planificación urbana. Ejemplifica tres orientaciones cardinales del eje de ordenación espacial: norte-sur, este oeste y noreste-suroeste; modelando volúmenes y cumbreras que garanticen disponer de luz solar no solo en un edificio singular sino también en los edificios vecinos (Knowles, 1981; 1974). Los estudios de este autor son cualitativos sin llegar a cuantificar las energías disponibles para uso humano, pero si realiza modelaciones volumétricas en manzanas para aplicar a la planificación urbana en California.

En esta línea, otros autores remarcan la necesidad del ser humano por recibir luz solar para satisfacer sus necesidades vitales, como el buen funcionamiento del ritmo circadiano. El cuerpo humano libera una hormona denominada melatonina que actúa como inductora del sueño y en consecuencia regula el metabolismo humano durante el día y la noche, también se le conoce como el reloj biológico (Turek et al., 2001:3).

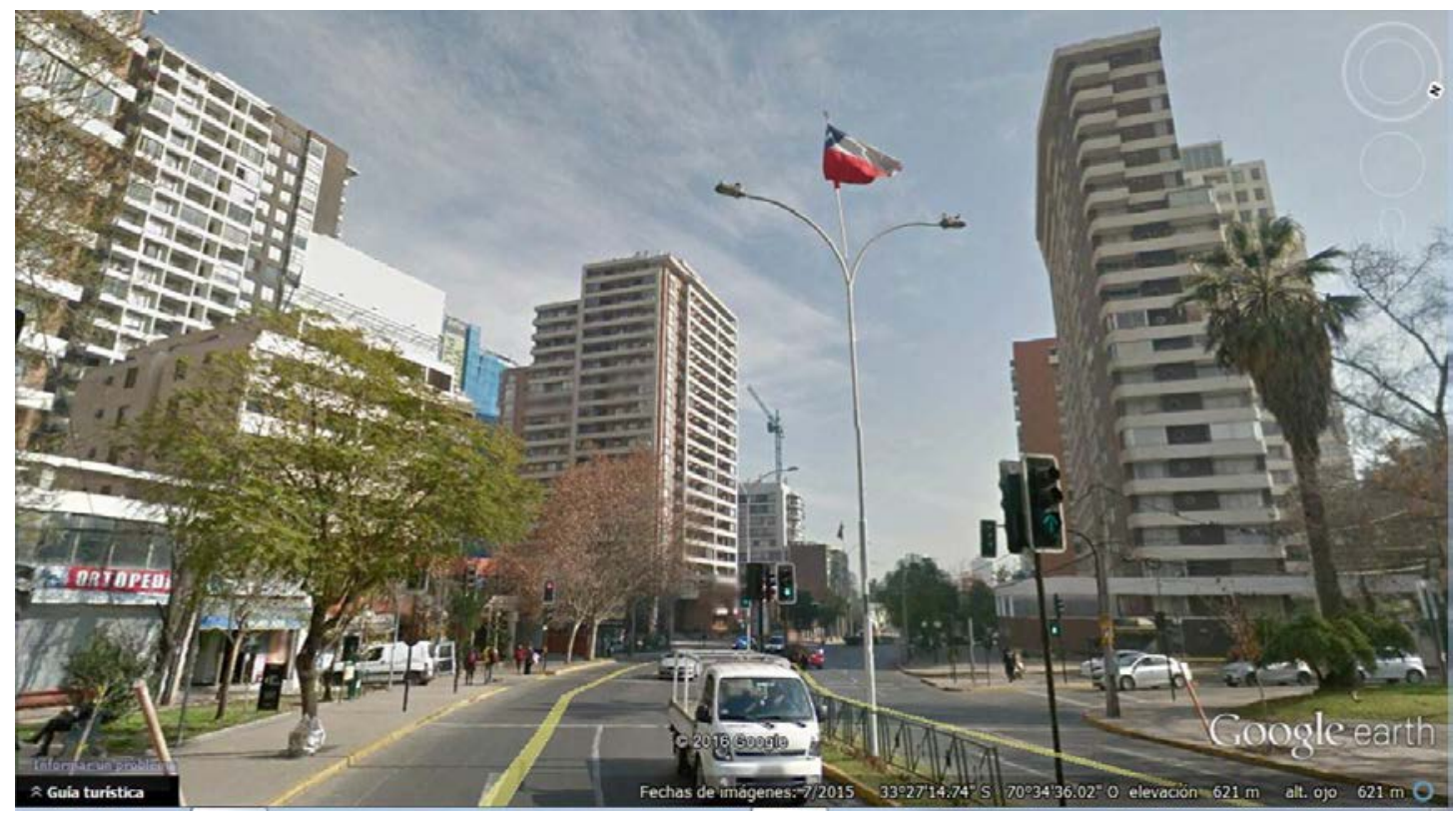

Fig. 1 Concentraciones inmobiliarias de gran altura que irrumpen en la morfología urbana pre existente. Fuente: Google Earth 


\section{Planificación urbana, norma urbanística y energética chilena asociada al inmobiliario residencial.}

Si bien la luz solar esta resguardada en la legislación chilena, ( MINVU, 2015) no es evidente su incorporación en la norma urbanística respecto a la forma de ocupación de suelos. En efecto, la Ordenanza General de Urbanismo y Construcción establece conceptos asociados tales como los distanciamientos, rasantes, sombras, forma arquitectónica, pero no explicita la variable energética solar como un bien a resguardar en el emplazamiento de los edificios. ${ }^{2}$ (OGUC 2015, PLANIF 6-4 a 6-11).

La agenda política de energía chilena establece entre uno de sus ejes el desarrollo de recursos energéticos propios (eje 3) y un sector energético eficiente en la gestión del consumo (eje 5). (Min. Energía, 2014: 43-47-48-65-67). Esto induce a pensar en la revalorización de la arquitectura y el urbanismo solar como un aporte desde la disciplina. (Szokolay, 1983: 1-6); (European Comission, 2010: 6-7). En efecto, entre estos ejes de la agenda se definen líneas de acción y metas tales como estimular la integración de las ERNC concordante con la Ley de Fomento de las ERNC (20/25); el desarrollo de un mercado de las ERNC de autoconsumo socialmente eficiente; Eficiencia Energética como Política de Estado y medidas para masificar los proyectos de eficiencia energética.

En la actualidad se observa que la aplicación de proyectos con Net Billing en Chile es ya una realidad aunque esté en su fase inicial y presente aún dificultades propias de la inexperiencia; enmarcadas en la Ley 20.571 de Generación Distribuida. (MINENERGIA, 2015). Los subsidios a la implementación de colectores solares en viviendas sociales durante el período 2010 a 2013 activaron la implementación de sistemas solares térmicos en viviendas nuevas unifamiliares, en la escala de barrios. Iniciativa que acaba de repetirse con la renovación de la franquicia tributaria otorgada por la Ley $\mathrm{N}^{\circ} 20.365$, extendiendo así su vigencia al 31 dic 2020 (DO. 05/02/2016). Esta ley focaliza en dos ejes: calentamiento de agua caliente para viviendas nuevas (franquicia a las constructoras) y viviendas sociales (subsidio a familias canalizada a través de las empresas) (BIT, 2016:94). Por otro lado, la Corporación de Desarrollo Tecnológico, organismo dependiente de la Cámara de la Construcción ha desarrollado desde el año 2010 documentos de apoyo para la implementación de sistemas solares térmicos y fotovoltaicos, y más recientemente manuales para el diseño y dimensionamiento de sistemas conectados a red (CDT, 2014:15). La venta de viviendas de sectores de ingresos medios en edificios de media altura, que ofrecían sistemas solares térmicos y reducción en costos de operación, para disponer de agua caliente sanitaria, fue un plus que actuó positivamente en la velocidad de venta de las unidades residenciales. Edificios aislados de gran altura que buscaban liderar el diseño ambiental y energético fueron enarbolados como estándares por la innovación tecnológica y el ahorro en los costos de energía de los usuarios (PORTAL INMOBILIARIO, 2008).

Lo anterior ratifica la necesidad de velar por las condiciones de forma urbana que permitan llegar la luz solar a las edificaciones vecinas y con ello asegurar la posibilidad de emplear energías renovables no convencionales (ERNC), mediante sistemas pasivos o activos, para así reducir indirectamente los consumos energéticos y/o generar energía limpia mediante nuevas tecnologías y redes de abastecimiento en la ciudad.

\footnotetext{
${ }^{2} \mathrm{Art}^{\circ} 2.6 .3$ sobre rasantes y distanciamientos. Art ${ }^{\circ} 2.6 .12$ y Art $^{\circ} 2.6 .13$ para cálculos de sombra sobre plano horizontal y 2.6.14 representación gráfica de las sombras del volumen, en Capítulo 6, Titulo 2: De IaPlanificación
} 

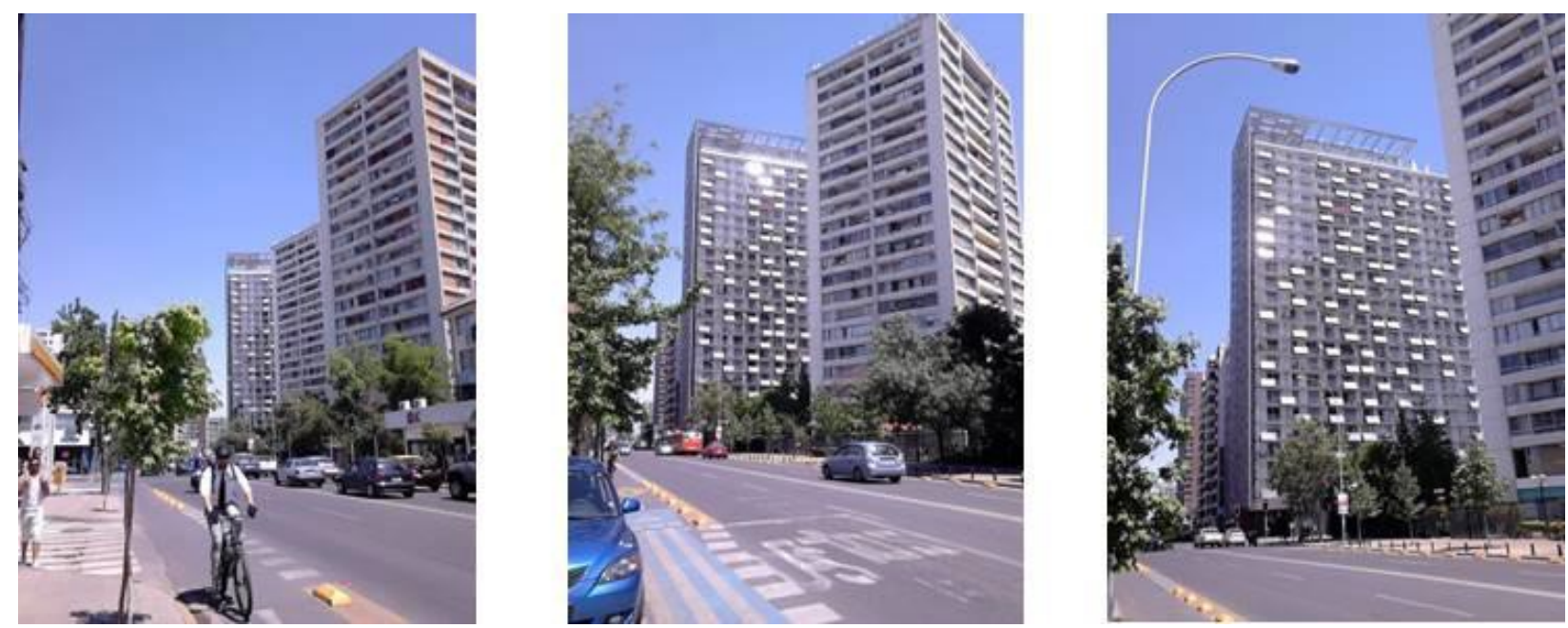

Fig. 2 Secuencia de acercamiento al Edificio GEN que incorpora paneles solares en fachadas Fuente: archivo del autor. Edificio GEN, Arquitecto F. Assadi

Desde una perspectiva teórica, Dupuy afirmaba que la red como concepto reorganiza la relación del hombre con el espacio y su territorio. Los flujos de materia, energía e información ocurren en un espacio de redes que los urbanistas aún no conciben en sus planes estáticos de regulación urbana (Dupuy, 1998:17-21). Esta postura consistía en un llamado de atención de una tendencia explosiva con el cambio de siglo, enfrentada a la sociedad del conocimiento y las tecnologías de información y comunicaciones (TICS). La ciudad del siglo XXI se ve enfrentada a una nueva forma de organización en base a conexiones digitales globales, o mundo virtual, que repercute radicalmente en la forma de vida y de organización espacial. En este sentido, Mitchell abogaba por una ciudad que funcione con sistemas inteligentes. (Mitchell, 2001: 19-21). Adicionalmente, Dyson con una visión futurista afirmaba que tres tecnologías revolucionaran el siglo XXI: el sol, el genoma y la internet alterando las convenciones éticas y sociales (Dyson, 2000).

Desde una perspectiva más práctica, el contexto chileno indica claramente actividad en el empleo de energía solar térmica y fotovoltaica para usos en edificios; tanto en política pública como en proyectos inmobiliarios en el último quinquenio. No obstante lo anterior, la pregunta que subyace es: ¿cómo regular el crecimiento de la ciudad?, para continuar con iniciativas de esta naturaleza, si la construcción en altura de un edificio vecino nuevo podría obstaculizar la captación de luz solar y con ello arruinar la inversión de un sistema solar o bien inhibir su futura instalación. En otras palabras, ¿Cómo resguardar mínimas condiciones para disponer de luz solar que alimente a los sistemas solares, pasivos o activos?

La planificación urbana establece parámetros generales y específicos para urbanizar los terrenos y edificar en la ciudad, influyendo de esta manera en la forma de la ciudad. (Ley OGUC, 2015). Los planes reguladores determinan áreas con distintas densidades urbanas otorgando variables prescriptivas a las edificaciones y a su emplazamiento en el predio: altura de edificación, coeficientes de ocupación de suelo (COS), coeficientes de constructibilidad, etc. Con ello, la arquitectura conjuga alternativas de configuración espacial, ya sea centradas en el espacio privado, y maximizando la rentabilidad de la inversión o también sintonizadas con el espacio público, en dialogo espacial con los edificios vecinos. Esta investigación no pretende dicotomizar visiones entre favorecer el espacio privado o el espacio público. El objetivo es buscar formas que combinen de una mejor manera los intereses divergentes de los actores en la ciudad. Por un lado, están los intereses privados, y por otro, los intereses de bien común y 
calidad de vida de los ciudadanos, que han de articularse de mejor manera en el espacio de la ciudad.

Con este objetivo, se exploran alternativas de intervención urbanística mediante modelos urbanos básicos de desarrollo inmobiliario en una manzana y simulaciones energéticas, de manera de exponer la importancia que tiene la relación entre forma urbana, energía y planificación ambiental. En particular, comprender que las intervenciones inmobiliarias sobre el tejido urbano de la ciudad repercuten en la disponibilidad de radiación solar en los edificios y suelos. Esto conduce a una exploración de alternativas de ocupación de suelo orientadas a conjugar ambos intereses, propios de la realidad urbana; la captación solar para bienestar humano junto con la maximización de una ocupación inmobiliaria.

Sanaieian realza la importancia que tiene el estudio de la manzana urbana en su forma y posición, pues no solo influencia el microclima en la ciudad, sino que el desempeño energético de la misma, y por tanto no tiene sentido considerar el edificio sin sus alrededores. (Sanaieian et al., 2014) El autor analiza in extenso el impacto de la forma de la manzana urbana en el desempeño ambiental de los edificios y su ambiente inmediato, categorizando en tres grupos los efectos: comportamiento térmico, acceso solar y ventilación natural dentro y fuera de los edificios.

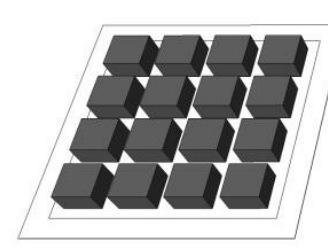

(a)

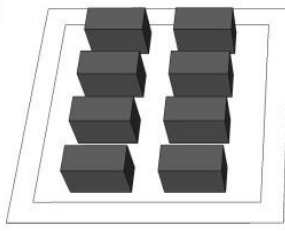

(b)

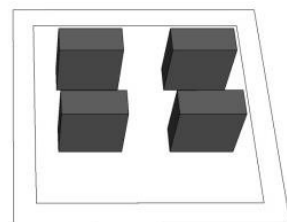

(c)

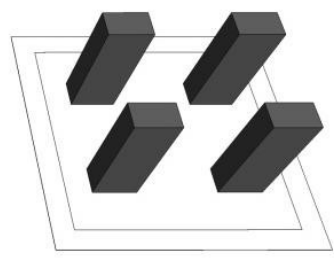

(d)

Fig. 3 Configuraciones espaciales según tipologías de edificación en altura para una manzana tipo e igual constructibilidad. (a)COS 0,47 y altura 4 pisos; (b) COS de 0,28 y altura 8 pisos; (c) $\cos 0,17$ y altura 12 pisos (d) COS 0,10 y altura 20 pisos

Fuente: Elaboración propia.

Tabla 1 Comparación de configuraciones espaciales según tipologías

\begin{tabular}{|c|c|c|c|}
\hline $\begin{array}{l}\text { Tipologías edificatorias } \\
\text { en tejidos urbanos }\end{array}$ & $\begin{array}{l}\text { Altura edificación } \\
\text { ( } N^{\circ} \text { pisos) }\end{array}$ & $\begin{array}{l}\text { Constructibilidad } \\
\left(\mathrm{N}^{\circ}\right)\end{array}$ & $\begin{array}{l}\text { Código } \\
\text { I dentificación }\end{array}$ \\
\hline Bloques de Vivienda & 4 & 2 & (a) \\
\hline Bloques de vivienda & 8 & 2 & (b) \\
\hline Torres de vivienda & 12 & 2 & (c) \\
\hline Torres de vivienda & 20 & 2 & (d) \\
\hline
\end{tabular}



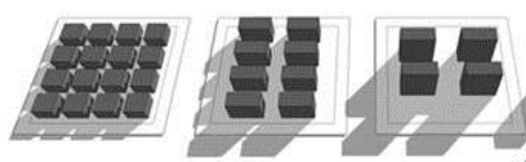

Santíago

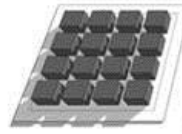

Valparaíso
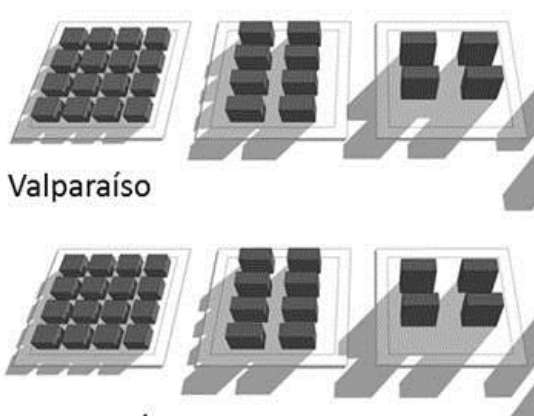

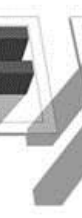

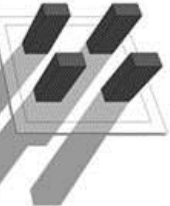

Concepción
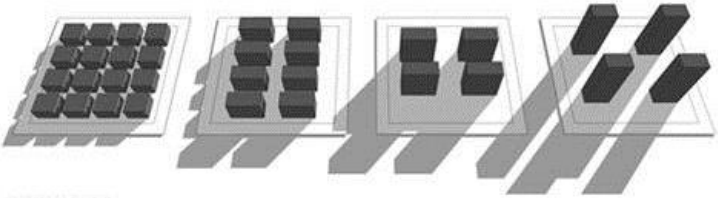

Temuco

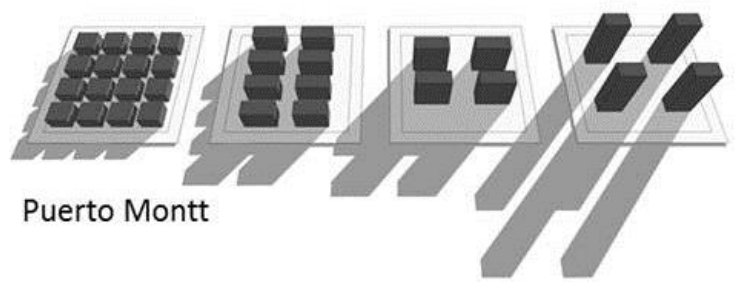

Fig. 4 Sombras arrojadas a las 0900hrs sobre suelos y fachadas de edificios vecinos en cinco ciudades chilenas. J ulio (invierno)

Fuente: Elaboración propia

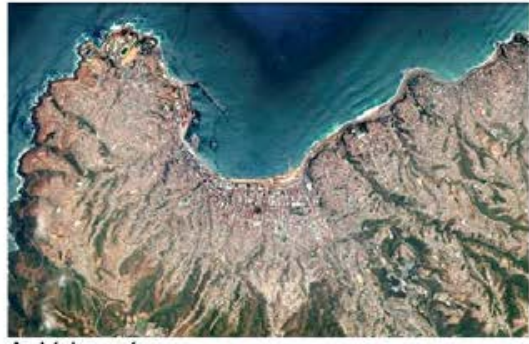

A. Valparaiso

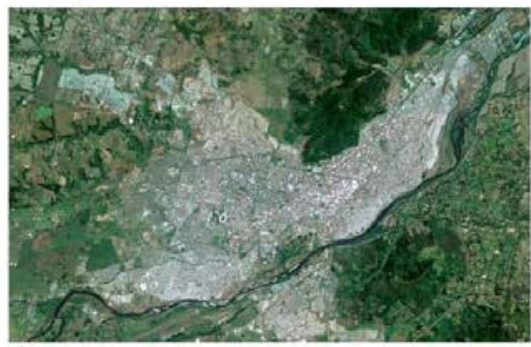

D. Temuco

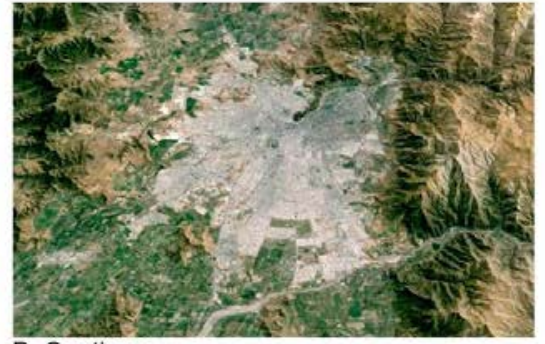

B. Santiago

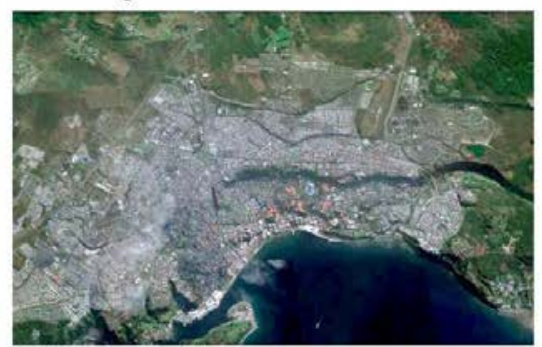

E. Puerto Montt

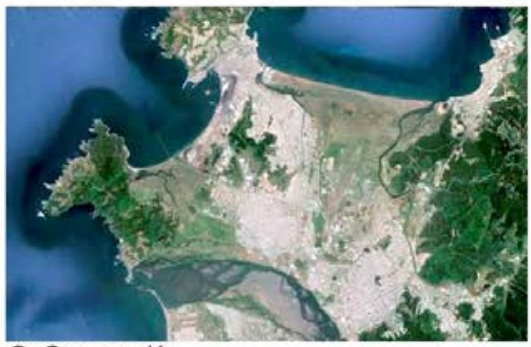

C. Concepción

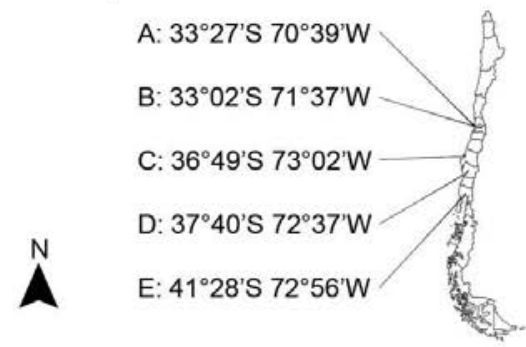

Fig. 5 Silueta urbanizada y entorno geográfico de cinco ciudades chilenas.

Fuente: Elaboración propia en base a Google Earth

\section{Metodología}

Dada la naturaleza del problema investigado, la aproximación metodológica es analítica y empírica para estudiar la relación entre variables, en este caso, la altura edificada permitida por una normativa urbanística y la energía disponible en suelos y fachadas (Kerlinger, 2002: 21). Los métodos empleados fueron la observación in situ, el modelamiento espacial 3D y la simulación energética. La observación in situ corresponde a la percepción del problema observado en ciudades chilenas que presentan niveles de urbanización creciente y desarrollos 
inmobiliarios de alta densidad. Las cinco ciudades chilenas seleccionadas se sitúan en el centro sur de Chile y son las siguientes: Santiago, Valparaíso, Concepción, Temuco y Puerto Montt. Estas ciudades fueron seleccionadas considerando sus niveles relativos más altos de urbanización respecto a otras y su localización espacial en el territorio que muestra una diferencia en el emplazamiento geográfico, pues se ubican en el borde costero y en el valle central. Con ello, es posible analizar la disponibilidad de la luz solar según latitud, clima y geografía.

Considerando la extensión latitudinal del territorio chileno, y la densidad edificatoria alcanzada por los desarrollos inmobiliarios, se propuso una configuración espacial con un coeficiente de constructibilidad medio-alto, encontrado en los planes reguladores vigentes. (IMLC, 1994;) Para ello, se construyeron modelos espaciales 3D mediante AutoCAD 2013, que representen tipologías morfológicas de ocupación de suelo para una manzana de 1ha., y un tamaño promedio en el centro de estas ciudades, la cual se empleó como modelo base. A partir de ella, se construyeron con el software SKETCHUP cuatro tipologías morfológicas de edificación en altura: bloques de vivienda de baja altura (4 pisos), bloques de vivienda de media altura ( 8 pisos), torres de vivienda de media altura ( 12 pisos), y torres de vivienda de gran altura (20 pisos), manteniendo constante una constructibilidad ${ }^{3}$ de $2^{4}$, pero variando la altura (número de pisos) y la ocupación de suelo (COS: coeficiente de ocupación de suelo). ${ }^{5}$ Fig. 3 y Tabla 1. En la Fig. 3 se indican las configuraciones: (a) el COS es de 0.47 y la altura es de 4 pisos; en (b) el COS es de 0,28 y la altura es de 8 pisos; en (c) el COS es de 0,17 y la altura es de 12 pisos; y finalmente en (d), el COS es de 0,1 y la altura es de 20 pisos.

Estas formas alternativas de ocupación del espacio en un tejido urbano tienen implicancias sobre el sombreamiento y la posibilidad de disponer de luz solar en los espacios habitados, sean públicos o privados. En la Fig. 4 se indican las sombras arrojadas por los edificios entre ellos y los suelos afectados temprano en la mañana, (0900hrs) para cinco ciudades en el mes de Julio, que corresponde a un mes frío y de mayor demanda energética. (Branth, 2013; UNTEC, 2014:12) Se observa que a medida que aumenta la altura edificada, la sombra afecta solo una fracción del suelo y edificios vecinos, en cambio a medida que la altura disminuye, cubre totalmente los espacios intersticiales entre edificios. Por tanto, una primera conclusión en base al modelo propuesto y período observado, es que a mayor altura y menor coeficiente de ocupación de suelo, el espacio urbano es menos afectado por las sombras. (Comparando (a) con $(d))$. En cambio, a menor altura y mayor coeficiente de ocupación de suelo, la afectación ocurre en ambos: tanto en el espacio urbano como en las edificaciones. En consecuencia, manteniendo el mismo coeficiente de constructibilidad, pero alterando la ocupación de suelo (COS) y la altura $(\mathrm{H})$, se presenta visualmente más beneficioso la captación de luz solar para manzanas con torres que manzanas con edificios baja altura, casi completamente sombreados. Es importante mencionar que dado que el modelo básico ha sido una manzana de 1 ha como punto de partida, los resultados no serán aplicables a un tejido urbano que es más complejo.

Posteriormente, se realiza una simulación energética al mismo modelo, con software Heliodon $2.6^{6}$ aplicado a las cinco ciudades chilenas considerando luz solar incidente sobre fachadas y

3 Coeficiente de Constructibilidad: número que multiplicado por la superficie total del predio, descontada de estas últimas las áreas declaradas de utilidad pública, fija el máximo de metros cuadrados posibles de construir sobre el terreno. Titulo1. Disposiciones generales. Art ${ }^{\circ} 1.1 .2$ OGUC

4 Se asume un predio de $10000 \mathrm{~m} 2$ factible a un desarrollo inmobiliario y una constructibilidad de 2, obtenida como un valor medio alto que solicitan los planes reguladores en algunas comunas chilenas

5 Coeficiente de Ocupación de Suelo: número que multiplicado por la superficie total del predio, descontadas de esta última las áreas declaradas de utilidad pública, fija el máximo de metros cuadrados posibles de construir en el nivel de primer piso. Titulo1. Disposiciones generales. Art ${ }^{\circ} 1.1 .2$ OGUC

6 Este software requiere integrar ulteriormente las condiciones climáticas locales, en particular los días de cielo claro y nubosidad predominante en la ciudad, porque estos modifican los valores obtenidos en la tabla. Este artículo es una 
suelos (Fig. 6). La elección de estos elementos de análisis se fundamenta en la importancia que ellos tienen en el urbanismo y en el desarrollo inmobiliario. En el desarrollo inmobiliario la variable suelo es relevante por la incidencia en los costos de inversión, (variable económica); en cambio las fachadas de muros son importantes para incorporar energías limpias en base a sistemas solares térmicos o fotovoltaicos, particularmente en el centro sur de Chile (variable tecnológica). Esta peculiaridad es relevante toda vez que la mayoría de las inversiones se localizan actualmente en el Norte Grande de Chile, dada la mayor intensidad de la radiación solar a escala mundial. No obstante, el centro del país tiene también un potencial muy interesante de explorar y explotar, dada la mayor cantidad relativa de energía solar disponible en fachadas sobre techumbres, durante el período de invierno ${ }^{7}$, por ubicarse en latitudes más altas de zonas climáticas templadas. (Cárdenas e Higueras, 2015: 217-237)

En el urbanismo, adquieren importancia ambos: las fachadas y los suelos; las fachadas constituyen el envolvente fundamental de la arquitectura y el medio de interacción térmica y lumínica con el entorno urbano o ambiente construido, lo cual incide en el confort y en la arquitectura bioclimática. (Neila, 2004) Los suelos cumplen una función fundamental, es el soporte del espacio urbano, y parafraseando a Bruno Secchi ..."donde se está en público"... (Schlak, 2007), junto con las fachadas configuran el espacio de la vida urbana. La morfología resultante del espacio y arquitectura modelan finalmente el espacio de la ciudad, la forma urbis, desde la perspectiva proyectual. (Gravagnuolo, 1998: 7)

A partir de las simulaciones energéticas presentadas en la Fig. 6, se confeccionan la Fig. 7, Fig. 8 y Fig. 9 que muestran la energía total disponible en el mes de Julio (invierno) y la energía disponible en fachadas y suelos respectivamente, por unidad de superficie durante 21 de Julio, para cada ciudad.

Para terminar el análisis de luz solar incidente en modelos morfológicos de desarrollo inmobiliario, acorde a norma urbanística, y en el contexto político actual de Net Billing; se realiza un cruce entre la disponibilidad energética en fachadas afectadas por sombras (situación más adversa) o potencia, y el consumo energético residencial promedio de una vivienda chilena. (Fig. 10). De esa manera, contrastar si la oferta disponible es suficiente para satisfacer el consumo requerido. Con ello, presentar de un modo preliminar la inicial discusión entre la morfología urbana resultante de un desarrollo inmobiliario, el marco normativo de la planificación urbana y la necesidad de generar energías renovables no convencionales ERNC para el autoconsumo nacional.

\section{Simulación energética en cinco ciudades chilenas}

En la Fig. 6 se observa cualitativamente la irradiación incidente sobre las fachadas (paramentos verticales) y suelos (paramentos horizontales) para el mes de julio en cada una de las cinco ciudades estudiadas. Esta simulación considera solamente la radiación solar directa sobre los planos y representa un valor total acumulado, asumiendo una orientación norte del modelo.

En la Fig. 7 se indica cuantitativamente un resumen de la energía total disponible por ciudad y configuración morfológica. Se observa que a mayor altura edificada, expresada en número de pisos, hay una mayor disponibilidad de energía solar, porque hay más superficie disponible, sin obstrucciones solares y con ellas. En los suelos, también se presenta la misma situación: mientras menos ocupación de suelo y crecimiento en altura, se alcanza una mayor radiación

aproximación conceptual y de postura crítica frente a un mandato nacional emergente, para lo cual se emplean aproximaciones numéricas pero deben ajustarse con otros algoritmos si se busca precisión en un proyecto.

7 Período cuando más se requiere la solar térmica y fotovoltaica para calentamiento de agua e iluminación. 
solar relativa, con gradientes energéticos según sean los distanciamientos de los edificios entre sí. La comparación visual entre las ciudades indica que a mayor latitud, la radiación solar disponible es menor en suelos, pero relativamente mayor en las fachadas. Esto se explicaría por el ángulo de incidencia solar durante el invierno, a medida que asciende latitudinalmente es más baja la altura solar.

En la Fig. 8 se muestra una síntesis de la energía total promedio en un día ( 21 Julio) captada en las fachadas (muros), afectadas por sombras de los inmuebles vecinos, y para las distintas configuraciones morfológicas: bloques de vivienda baja altura, bloques de vivienda media altura, torres de vivienda media altura y torres de vivienda gran altura. Las curvas indican que a mayor latitud se dispone de menor energía, como es esperable una relación inversamente proporcional. Sin embargo, mientras mayor sea la altura edificada en cada configuración, se dispone de mayor potencia de energía en las fachadas, lo cual se explica por la mayor superficie disponible. Lo anterior sugiere edificar en altura si se quiere fomentar el uso de energía solar fotovoltaica en fachadas, lo cual corresponde al ámbito privado edilicio de la arquitectura.
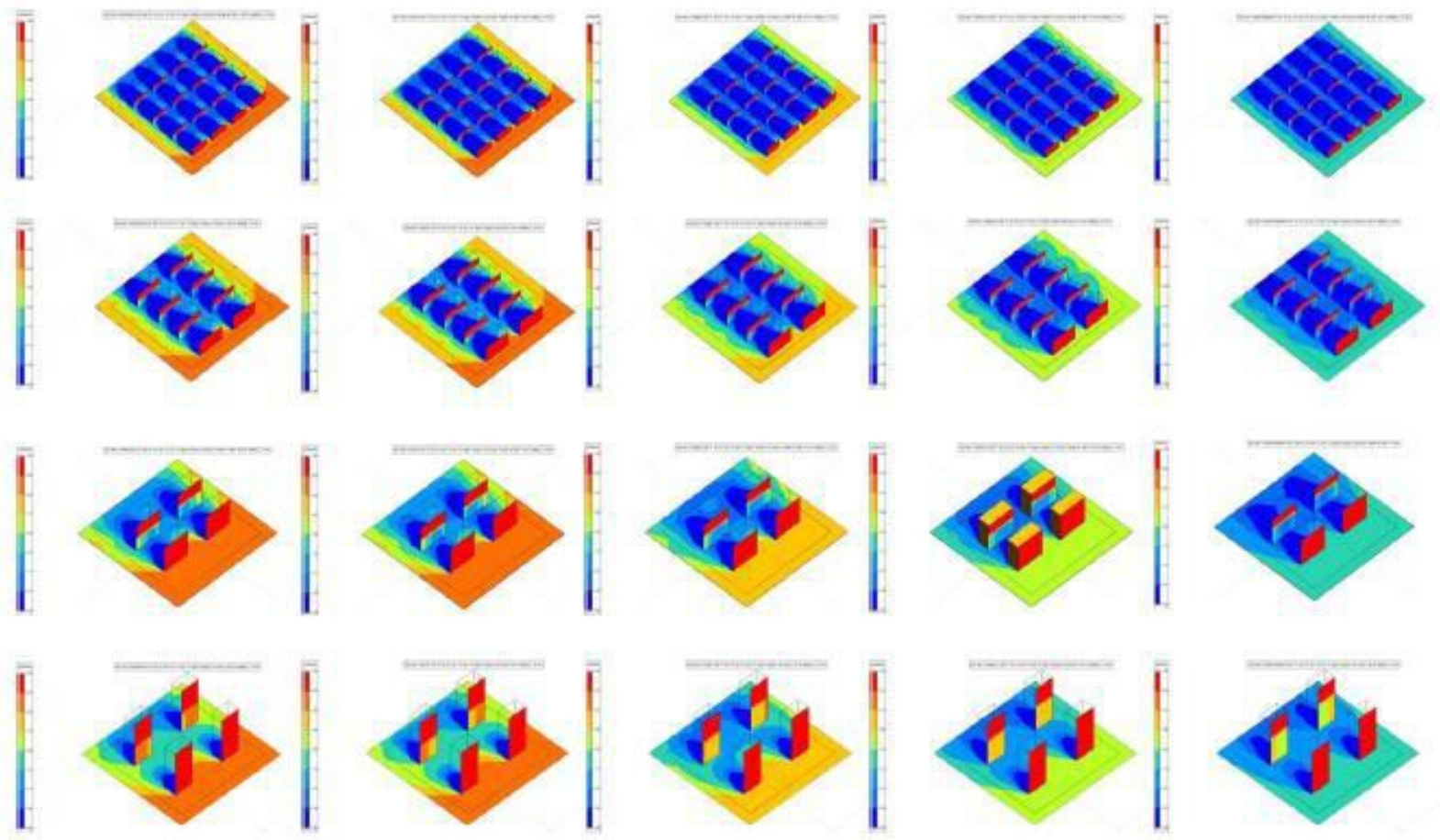

Fig. 6 Radiación solar directa en muros (paramentos verticales) y suelos (paramentos horizontales) de las configuraciones morfológicas en $\mathbf{5}$ ciudades chilenas.

Fuente: Elaboración propia 


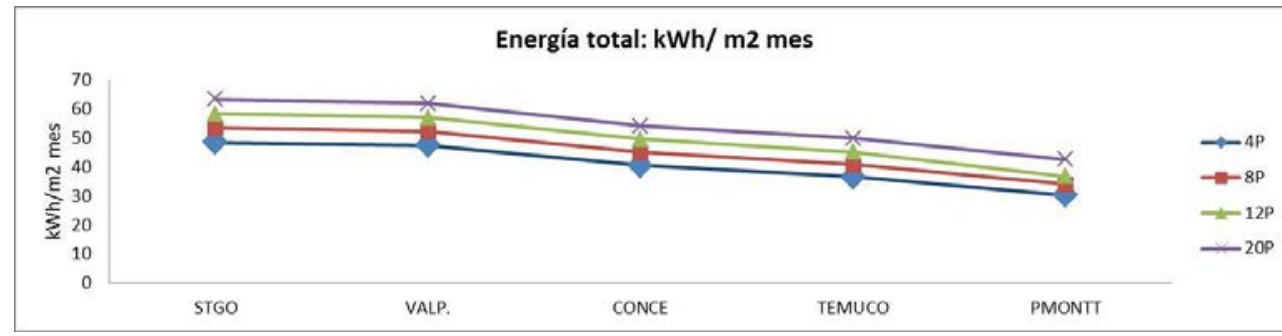

Fig. 7 Energía total disponible por ciudad y configuración morfológica, J ulio Fuente: Elaboración propia

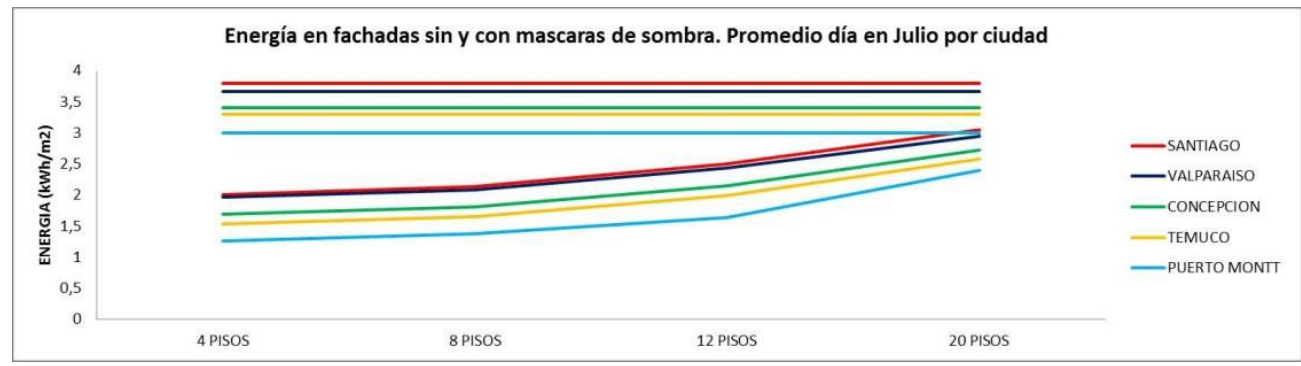

Fig. 8 Energía disponible en fachadas afectadas por sombras. Promedio día en J ulio por ciudad y configuración morfológica.

Fuente: Elaboración propia

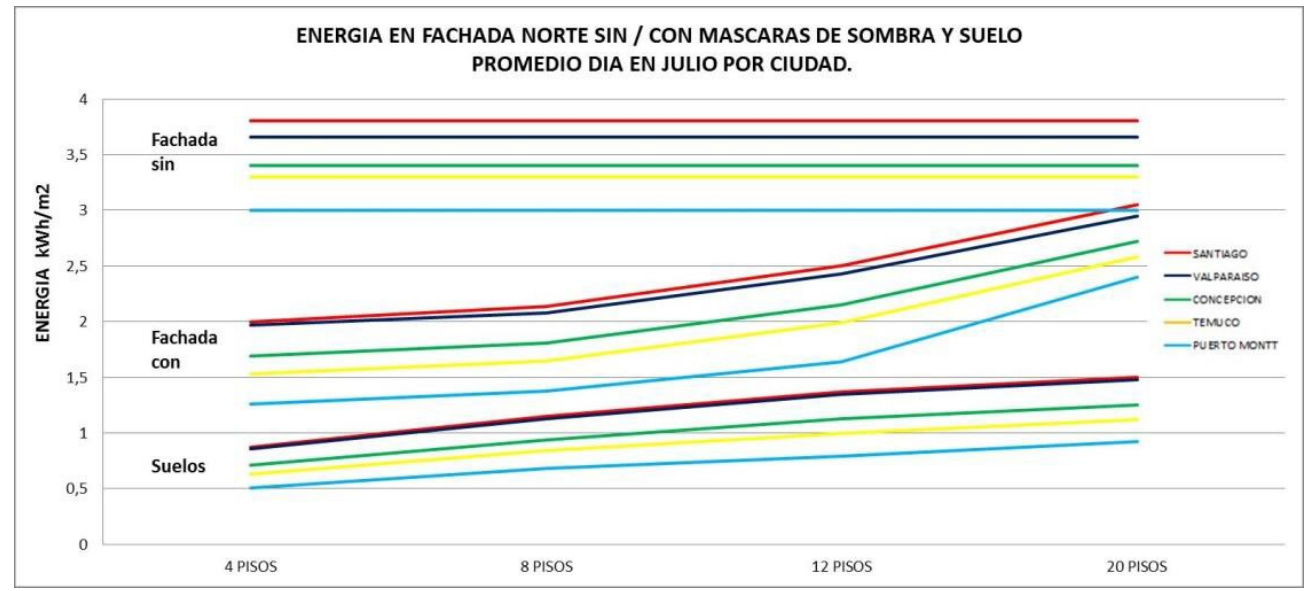

Fig. 9 Energía disponible en suelos afectados por sombras. Promedio día en Julio por ciudad y configuración morfológica.

Fuente: Elaboración propia

En la Fig. 9 se presenta una síntesis de la energía disponible promedio en un día (21 Julio), captada en los suelos y afectados por sombras de los inmuebles vecinos, para las distintas configuraciones morfológicas: bloques de vivienda baja altura, bloques de vivienda media altura, torres de vivienda media altura y torres de vivienda gran altura. Las curvas indican una relación inversa entre latitud y energía disponible para cada ciudad según la configuración morfológica. No obstante, nuevamente se observa una relación directamente proporcional entre la altura edificada y la oferta de energía solar. Si se quiere preservar energía solar en el dominio público (espacio urbano), sometido a coeficientes de constructibilidad alta, se sugiere incentivar dicha altura 
En la Tabla 2 se muestra una síntesis de los valores resultantes de la simulación energética en fachadas y suelos por configuración morfológica para el 21 de Julio. Estos valores indican que a medida que aumenta la altura se dispone de más energía sobre los suelos y las fachadas, aún en condiciones de enmascaramientos. Este hecho se explicaría porque se dispone de más superficie de muros para capturar el sol y fracciones de suelo que reciben la radiación solar directa.

Tabla 2 Comparación de energía promedio en fachadas y suelos para un día de Julio en cinco ciudades

\begin{tabular}{|c|c|c|c|c|c|c|c|c|c|c|}
\hline CONFIGURACIONES & FACHADA & FACHADA & FACHADA & FACHADA & FACHADA & & & & & \\
\hline MORFOLOGICAS EN & $\begin{array}{c}\text { ENMASCARAD } \\
\mathrm{A}\end{array}$ & $\begin{array}{c}\text { ENMASCARAD } \\
\text { A }\end{array}$ & $\begin{array}{c}\text { ENMASCARAD } \\
\text { A }\end{array}$ & $\begin{array}{c}\text { ENMASCARAD } \\
\text { A }\end{array}$ & $\begin{array}{c}\text { ENMASCARAD } \\
\mathrm{A}\end{array}$ & SUELO & SUELO & SUELO & SUELO & SUELO \\
\hline UNA MANZANA & SANTIAGO & VALPARAISO & CONCEPCION & TEMUCO & PUERTOMONTT & SANTIAGO & VALPARAISO & CONCEPCION & TEMUCO & PUERTOMONTT \\
\hline bloques vivienda baja altura & 2 & 1,97 & 1,69 & 1,53 & 1,26 & 0,87 & 0,86 & 0,71 & 0,63 & 0,51 \\
\hline bloques vivienda media & 2,14 & 2,08 & 1,81 & 1,65 & 1,38 & 1,15 & 1,13 & 0,94 & 0,84 & 0,68 \\
\hline torres vivienda media & 2,5 & 2,43 & 2,15 & 1,99 & 1,64 & 1,37 & 1,35 & 1,13 & 1 & 0,79 \\
\hline torres vivienda gran altura & 3,05 & 2,95 & 2,72 & 2,58 & 2,4 & 1,5 & 1,48 & 1,25 & 1,12 & 0,92 \\
\hline
\end{tabular}

Fuente: Elaboración propia

A partir de la Tabla 2 se confecciona la Fig. 10, que muestra un histograma de la oferta solar y consumo promedio de energía por concepto de iluminación, para cada ciudad y por configuración morfológica.

\section{Autosuficiencia energética y la Generación Distribuida}

El Net Billing es una medida política para fomentar la generación de energía fotovoltaica en base a ERNC, principalmente solar y se basa en la capacidad de cogeneración de las unidades residenciales hasta un máximo de $100 \mathrm{~kW}$ de energía solar fotovoltaica (electricidad). Con ello, permitir el autoconsumo y la factibilidad de inyectar a la red de distribución el excedente de energía. Esta situación requiere conocer cuál es la disponibilidad de energía in situ y si alcanza a cubrir la demanda residencial. Por ello, se analizó la energía disponible en fachadas afectadas por sombras en cada configuración morfológicas y por ciudad, junto con el consumo medio de una vivienda chilena de $100 \mathrm{~m}^{2}$ en cada una de las cinco ciudades. Para ello, se consideró un consumo promedio de $192 \mathrm{kWh} / \mathrm{m}^{2}$ año, como valor global total destinado a calefacción (80\%), agua caliente sanitaria [ACS] (19\%) e iluminación (1\%), según consta en los estudios de la Agencia Chilena de Eficiencia Energética (Brandt, 2013) y el Sistema de Calificación Energética de Viviendas (MINVU, 2015). Considerando que las simulaciones de oferta solar fueron realizadas para un día de invierno, entonces los valores de consumo se calculan también para un día solamente. Por tanto, el valor del consumo promedio anual se multiplica por $100 \mathrm{~m}^{2}$ y se divide en 365 días; obteniendo así un valor día de $52,6 \mathrm{kWh} / \mathrm{m}^{2} .{ }^{8}$ Luego, a ese valor se calcula el $1 \%$ correspondiente a iluminación (electricidad) y se obtiene un nuevo valor de 0,526 $\mathrm{kWh} / \mathrm{m}^{2}$

Una vez conocido este valor de consumo medio energético para una vivienda chilena se cruza con el valor de disponibilidad energética solar en una manzana edificada, obtenida de cada simulación en cada ciudad. Si se asume que en cada piso se construyen 2 viviendas y la configuración de bloques de baja altura tiene 4 pisos, entonces se obtiene un total de 8 viviendas, las cuales consumirían un total de $4,16 \mathrm{kWh} / \mathrm{m}^{2}$. En la figura 10 , se observa que la

8 Estos cálculos son aproximaciones al problema estudiado, pues requiere ajustes según la estación del año y localización geográfica. En invierno el consumo de iluminación es mayor que en verano y las ciudades sureñas disponen de menos luz solar porque la trayectoria del sol es más corta y su posición geográfica más tangencial. 
disponibilidad de energía solar (potencia) en condiciones de sombras (enmascaramientos) para un día de invierno, no son suficientes para satisfacer el consumo promedio de una vivienda chilena.

Ello plantea una discusión sobre la morfología urbana que optimice la captación solar, ya no solo para propiciar una arquitectura bioclimática sino también para generar energía solar que pueda satisfacer necesidades para el autoconsumo. El vínculo entre la planificación urbana, gestión energética y morfología de los desarrollos inmobiliarios es un camino apenas explorado en las ciudades chilenas que requiere ser investigado para fomentar integralmente las políticas públicas, en vez de hacerlo solo sectorialmente.

Investigaciones sobre instrumentos de control de forma urbana considerando la variable energética solar han sido desarrolladas también en otras ciudades, empleando proyecciones estereográficas como métodos de planificación urbana para medir desempeño de la estructura urbana en la insolación e iluminación del ambiente construido. (Ruttkay, Nome y Turkienitkz, 2001: 217). Investigaciones recientes señalan que las densidades de ocupación afectan el potencial solar barrios residenciales organizados en cluster. ( $\mathrm{Li}, 2015$ ). Búsquedas morfológicas en edificios residenciales considerando fachadas urbanas en bloques de manzanas que optimicen la captación solar están siendo investigados muy recientemente (Hachem et al., 2014). En Chile, aún no se inicia este proceso y se cree que esta investigación puede representar un punto de partida a la discusión sobre luz solar en la normativa urbana chilena y morfologías para los desarrollos inmobiliarios que optimicen su captación en los propios edificios como también en su entorno urbano.

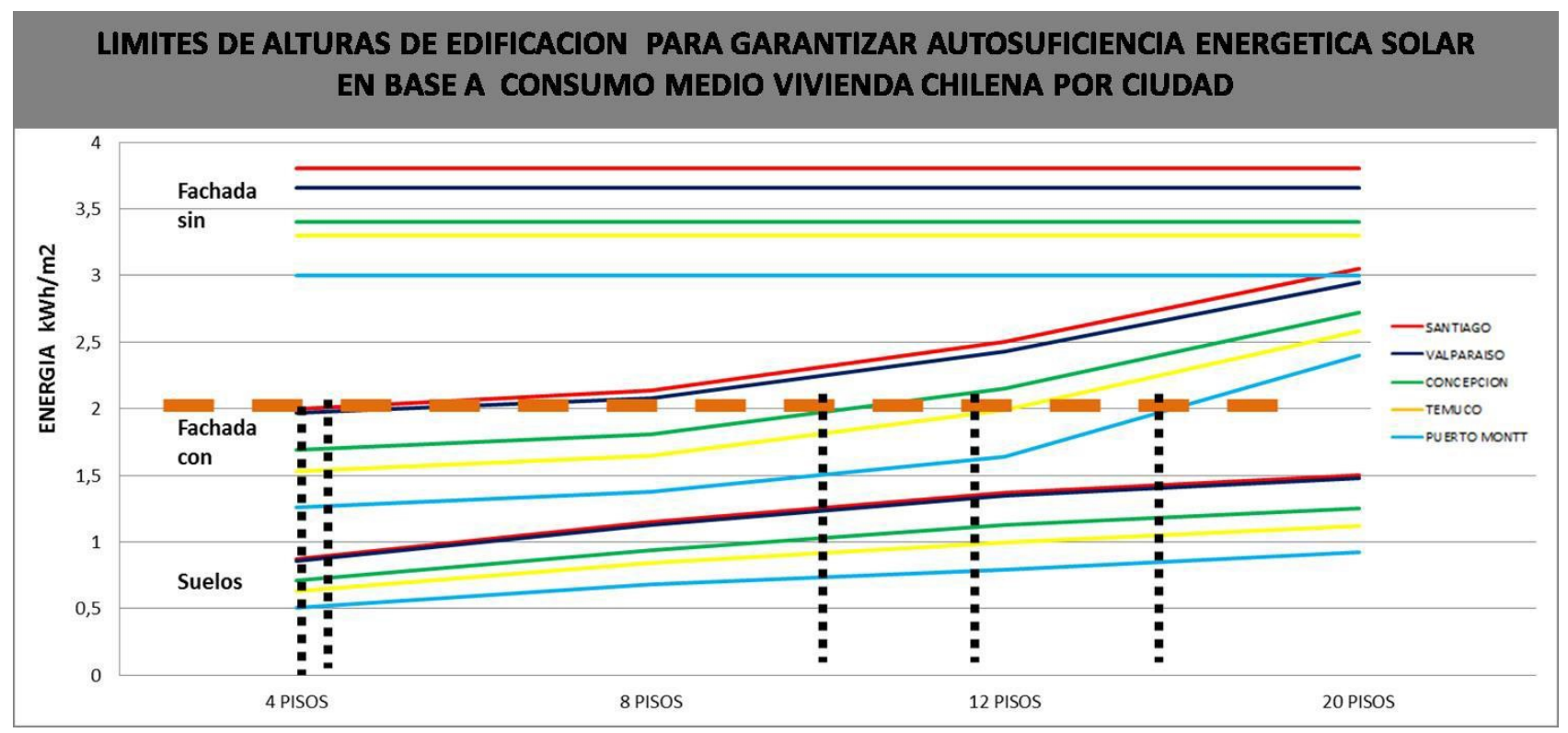

Fig. 10 Contraste entre oferta solar y consumo energía PV promedio para vivienda chilena en un día de Julio (invierno)

Fuente: Elaboración propia

\section{Discusión y conclusiones}

Una simulación energética solar sobre cuatro tipologías de configuración morfológica, empleadas en los desarrollos inmobiliarios, para cinco ciudades chilenas ha sido realizada como punto de partida para plantear una discusión sobre la relación entre planificación urbana, energética y formas de ocupación en manzanas urbanas. Discusión relevante en el contexto 
actual sobre Net Billing que permite inyectar el excedente de energía limpia generada por una unidad residencial hacia la red de distribución eléctrica.

Los parámetros de los instrumentos de planificación tales como coeficiente de ocupación de suelo, alturas edificadas y constructibilidad han sido modelados para estudiar la disponibilidad energética solar y su posible uso en autoconsumo, considerando a groso modo, la oferta solar y el consumo promedio de una vivienda chilena. Los resultados indican que a medida que aumenta la altura edificada en una manzana, aumenta la disponibilidad energética, tanto en fachadas (ámbito privado) como en los suelos (dominio público). Ello sugiere preliminarmente que la mayor altura edificada proporcionaría más energía que una configuración de baja altura para un día de invierno, en el mes de julio.

Adicionalmente, a medida que se asciende en latitud, se reduce la radiación solar directa en su totalidad, pero mejora en términos relativos su desempeño en fachadas respecto a los suelos, explicado por la menor altura solar en Puerto Montt respecto a Santiago y su ángulo de incidencia en fachadas.

Finalmente, se realiza un ejercicio de simulación entre la oferta disponible y el consumo promedio de una vivienda de $100 \mathrm{~m} 2$ en Chile, contrastando los valores obtenidos de la simulación y una base de datos del Ministerio de la Vivienda y Urbanismo; obteniendo la insuficiencia para el autoconsumo total. No obstante, una aplicación parcial podría tener cabida. Investigaciones de forma urbana asociada a la captación y optimización de luz solar son tareas pendientes a desarrollar por arquitectos, urbanistas y planificadores.

\section{Agradecimientos}

Este artículo es resultante del proyecto FONDECYT 1130139 - CONICYT Chile, y se agradece el financiamiento otorgado para su ejecución como también para la formación de tesistas; Srta. Claudia Acevedo Saavedra.

\section{Referencias}

Alcaino Vargas, J. (2008). Efectos de las Normas de Excepción sobre la Planificación Urbana Comunal y sobre los Conflictos Urbanos (Tesis de Magister en Desarrollo Urbano). Pontificia Universidad Católica de Chile, Santiago de Chile.

Branth, R. (2013). Calificación energética de viviendas. Recuperado de http://www.acee.cl/sites/default/files/noticias/documentos/Presentacion\% 20Ragnar\%20\%20Brant $\underline{\text { h.pdf }}$

Becar, P. (2013). Fusión de terrenos. Recuperado de https://www. youtube.com/watch?v=TLbEa1Tcrw8

BIT. (2016). Renovación de la franquicia tributaria para sistemas solares térmicos. Recuperado de http://www. revistabit.cl/revistabit/Uploads/107/63910101150005339094-t4.pdf

Cárdenas-Jiron, L. A. y Higueras, E. (2015). El barrio solar. Madrid: Editorial Mairea.

Cardenas-Jiron, L. A. y Vilches, D. (2015). Thermal comfort in housing under solar obstruction derived from building in urban renovation areas. 9th International Conference on urban Climate ICUC, Toulouse. 
Corporación de Desarrollo Tecnológico, CDT y Cámara Chilena de la Construcción, CChC. (2014). Diseño y dimensionamiento de sistemas solares fotovoltaicos conectados a red. Santiago: Autor.

Daher, A. (2013). El sector inmobiliario y las crisis económicas. EURE, 39(118). 47-76. https://doi.org/10.4067/s0250-71612013000300003

De Mattos, C.A. (2002). Transformación de las ciudades latinoamericanas. ¿lmpactos de la globalización? EURE, 28, 85. https://doi.org/10.4067/s0250-71612002008500001

Dupuy, G. (1998). El urbanismo de las redes. Teorías y métodos. Barcelona: Oikos-Tau.

Dyson, F. (2000). El sol, el genoma e internet. Las tres cosas que revolucionarán el siglo XXI: la energía solar, la ingeniería genética y la comunicación mundial. Madrid: Editorial Debate.

European Commission. (2010). Solar urban planning: Manual of best practices. Lisboa: POLIS

Galetovic, A. (Ed.). (2006). Santiago. Dónde estamos y hacia dónde vamos. Santiago de Chile: Centro de Estudios Públicos.

Gravagnuolo, B. (1998). Historia del urbanismo en Europa. Madrid: Ediciones Akal.

Hachem, C., Athienitis, A., \& Fazio, P. (2014). Energy performance enhancement in multistory residential buildings. Applied Energy, 116, 9-19. https://doi.org/10.1016/j.apenergy.2013.11.018

Knowles, R. (2003). The solar envelope: Its meaning for energy and buildings. Energy and Buildings, 35(1), 15-25. https://doi.org/10.1016/s0378-7788(02)00076-2

Kerlinger, F. (2002). Investigación del comportamiento. México, DF: Mc Grow-Hill.

Knowles, R. (1981). Sun rhythm form. Cambridge, MA: MIT Press.

Knowles, R. (1974). Energy and form. Cambridge, MA: MIT Press.

LI, D., Liu, G., \& Liao, S. (2015). Solar potential in urban residential building. Solar Energy, 111, 225235. https://doi.org/10.1016/j. solener.2014.10.045

Ministerio de Energía. (2014). Agenda de energía. Santiago de Chile: Autor.

Ministerio de Energía. (2015). Principales aspectos regulatorios de la Ley 20.571. Santiago de Chile: Autor.

Ministerio de Vivienda y Urbanismo, Minvu. (2015). Ley General de Urbanismo y Construcciones. D.F.L. N458 DE 1975. Recuperado de http://www.minvu.cl/opensite_20070404173759.aspx

Ministerio de Vivienda y Urbanismo, Minvu. (2015). Ordenanza General de Urbanismo y $\begin{array}{lllll}\text { Construcciones. } & \text { D.S.N }{ }^{\circ} 47 & \text { DE } & \text { 1992. }\end{array}$ http://www.minvu.cl/opensite_20070404173759.aspx

Ministerio de Vivienda y Urbanismo, Minvu. (2015). Sistema de Calificación Energética de Viviendas. Recuperado de http://calificacionenergetica.minvu.cl/media/Presentaci\%C3\%B3nCalificaci\% C3\% B3n-Energ\% C3\% A9tica-de-Viviendas-Abril-2015.pdf 
Mitchell, W. J. (2002). E-topías. Barcelona: Gustavo Gili.

Monette, F. \& Becker, B. (2014). Solar potential and solar impact (1 1 a edition). London: Wiley.

Neila, F. J. (2004). Arquitectura bioclimática en un entorno sostenible. Madrid: Munilla Leiría.

Portal Inmobiliario. (2008). Edificio GEN: Una nueva generación de edificios. Recuperado de http://www.portalinmobiliario.com/diario/noticia.asp?Noticial D $=8193$

Ratti, C., Baker, N., \& Steemers, K. (2005). Energy consumption and urban texture. Energy and Building, 37(7), 762-776. https://doi.org/10.1016/j.enbuild.2004.10.010

Ruttkay, F., Nome, A., \& Turkienikz, B. (2001). A methodology sunlight urban planning: A computerbased solar and sky vault obstructions analysis. Solar Energy, 70(3), 217-226. https://doi.org/10.1016/s0038-092x(00)00094-3

Sanaieian, H., Tenpierik, M., Van Der Linden, K., Mehdizadeh Seraj, F. \& Mofidi Shemrani, S. (2014). Review of the impact of urban block form on thermal performance, solar access and ventilation. Renewable and Sustainable Energy Reviews, 38, 551-560. https://doi.org/10.1016/j.rser.2014.06.007

SEC. (2014). Genera tu propia energía. Recuperado de http: //www.sec.cl/portal/page?_pageid=33,5819695\&_dad=portal\&_schema=PORTAL

Schlak, E. (2007). Espacio público. Revista ARQ, 65, 25-27. https://doi.org/10.4067/s0717$\underline{69962007000100006}$

Szokolay, S. V. (1983). Arquitectura solar. Barcelona: Editorial Blume.

Takahashi, J., Turek, F., \& Moore, R. (Ed.). (2001). Handbook of Behavioral Neurobiology. Springer Science-Business Media.

Untec. (2014). Alternativas tecnológicas para calefacción residencial con energías renovables no convencionales aplicables a la realidad chilena. Santiago de Chile: Subsecretaría del Medio Ambiente. 